\title{
Assessment and Prediction Planning of R.C Structures Using BIM Technology
}

\author{
${ }^{(1)}$ Nahla Ali Mohamed, ${ }^{(2)}$ Ahmed Mohammed Abdel-Alim , ${ }^{(3)}$ Hatem Hamdy Ghith,${ }^{(4)}$ Alaa \\ Gamal Sherif, \\ ${ }^{1}$ Assistant Lecturer at Housing and Building National Research Center, Cairo, Egypt. \\ Email:Nahlaali_hbrc11@yahoo.com \\ ${ }^{2}$ Associate Professor of Project Management, faculty of engineering at Mataria, Helwan \\ University, Cairo, Egypt. \\ ${ }^{3}$ Professor of R.C Structure Housing and Building National Research Center, Cairo, Egypt \\ ${ }^{4}$ Professor of R.C Structures, faculty of engineering at Mataria, Helwan University, Cairo, Egypt.
}

\begin{abstract}
Using Building information modeling (BIM) is very useful to facilitate the condition assessment of the building and record all rehabilitation work that has taken place during its life cycle through the building life cycle. The asset owners can receive the benefit of using BIM by incorporating the principles of BIM into the operation and maintenance of buildings. The main focus is on the integration of maintenance data by using building information modeling (BIM) to facilitate efficient inspection planning and to improve the condition assessment process for building elements. This paper presents a framework based on BIM for buildings condition assessment. The framework consists of two models: Condition Assessment Model and deterioration Predictive Model. The Condition Assessment Model manages the condition data that are collected during visual inspection. Integrated with failure records and life cycle prediction models, the deterioration predictive Model integrates with the Condition Assessment Model and forecasts elements failure. The results provide a basis for predictive maintenance of building elements. BIM is also adapted to manage and visualize not only the inspection data but also the predictive maintenance data.
\end{abstract}

Keywords: Condition Assessment, Building Information Modeling, surface condition, deterioration predictive model, Life cycle prediction, Maintenance action, Failure prediction.

\section{Introduction}

An attractive feature of BIM includes its potential for managing information throughout the entire life cycle of a structure. From early conceptual design to construction simulation, BIM is a proven and established tool. The Building information modeling for Inspection and Evaluation was developed to serve two purposes. First, it is used to demonstrate the capacity for BIM software to capture and reuse information gathered during an inspection to automate the structural evaluation process. Second, it is used to identify what is needed, what is available, and what is 
not yet available for a practical application of BIM into the building. However, little has been done to implement the use of BIM into the later stages of the project life cycle (Meadati, 2009). Building condition assessment concept via BIM Based on figure 1, the development of the BIM model and Building Condition Assessment works should be carried out simultaneously to achieve the good practice of building management for the existing building (Che-Ani et al., 2017).

According to the Smart Market Report 2012, almost 60\% of architecture and engineering firms are receiving the benefit of utilizing BIM for the design stages of a project, but only $29 \%$ of asset owners are receiving the benefit of utilizing BIM for the operation and maintenance stages (Construction, 2012).

One reason for this is the lack of as-built BIM models for structures requiring maintenance. In the bridge management field, creating individual models for each structure in an inventory would be untimely and economically unfeasible using conventional methods. With emerging technology, digitizing and modeling an entire bridge inventory could be practical for management purposes. For example, with the application of 3D laser scanning, a bridge could be modeled in a timely manner from dense point cloud data, which can capture both the detailed and precise geometry required (Tang and Akinci, 2012).

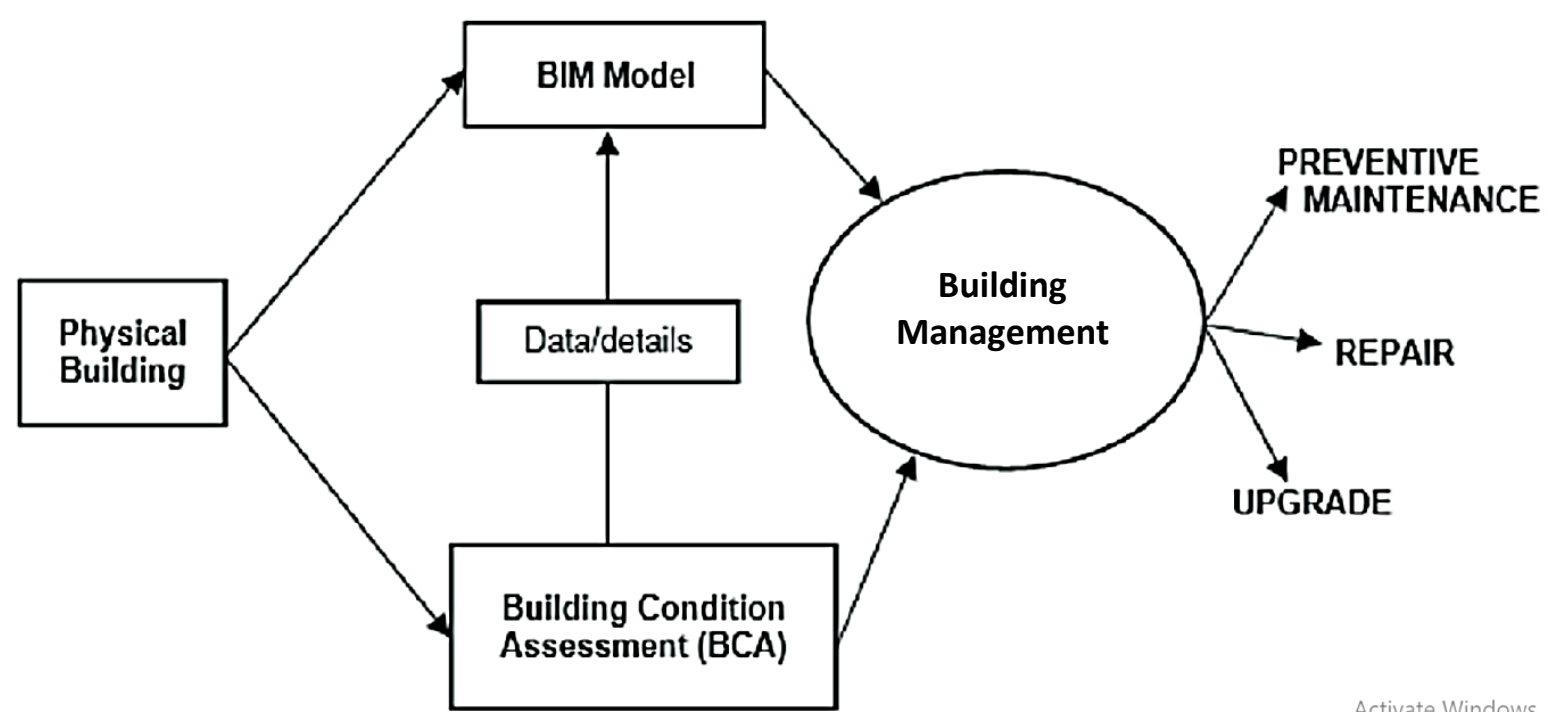

Figure 1: Building Condition Assessment Concept Via BIM (Che-Ani et al., 2017)

\section{Building Documentation Using Revit Structure}

The goal of the following section shows how the model can be used and offer additional insight on the benefit that decision makers might realize in utilizing BIM. As a visual tool, inspector could benefit in utilizing the Revit model because of its potential for accuracy and the level of details it could attain in identifying problem 
element. Information that is attached with the different building elements needs some ways to be displayed in a user friendly forms. This information includes physical description, and status of the building elements.

The Revit database developed for building model contains tables related to building elements (beams, columns and slabs). The Revit database contains the added inspection fields by this research shown in figure 2 .

In addition the model allows the user in the office to view all inspection data which record in field inspection such as pictures, text comments, and severities evaluations. Certainly, it is allowed to display all the data entered on the form after the building evaluation.

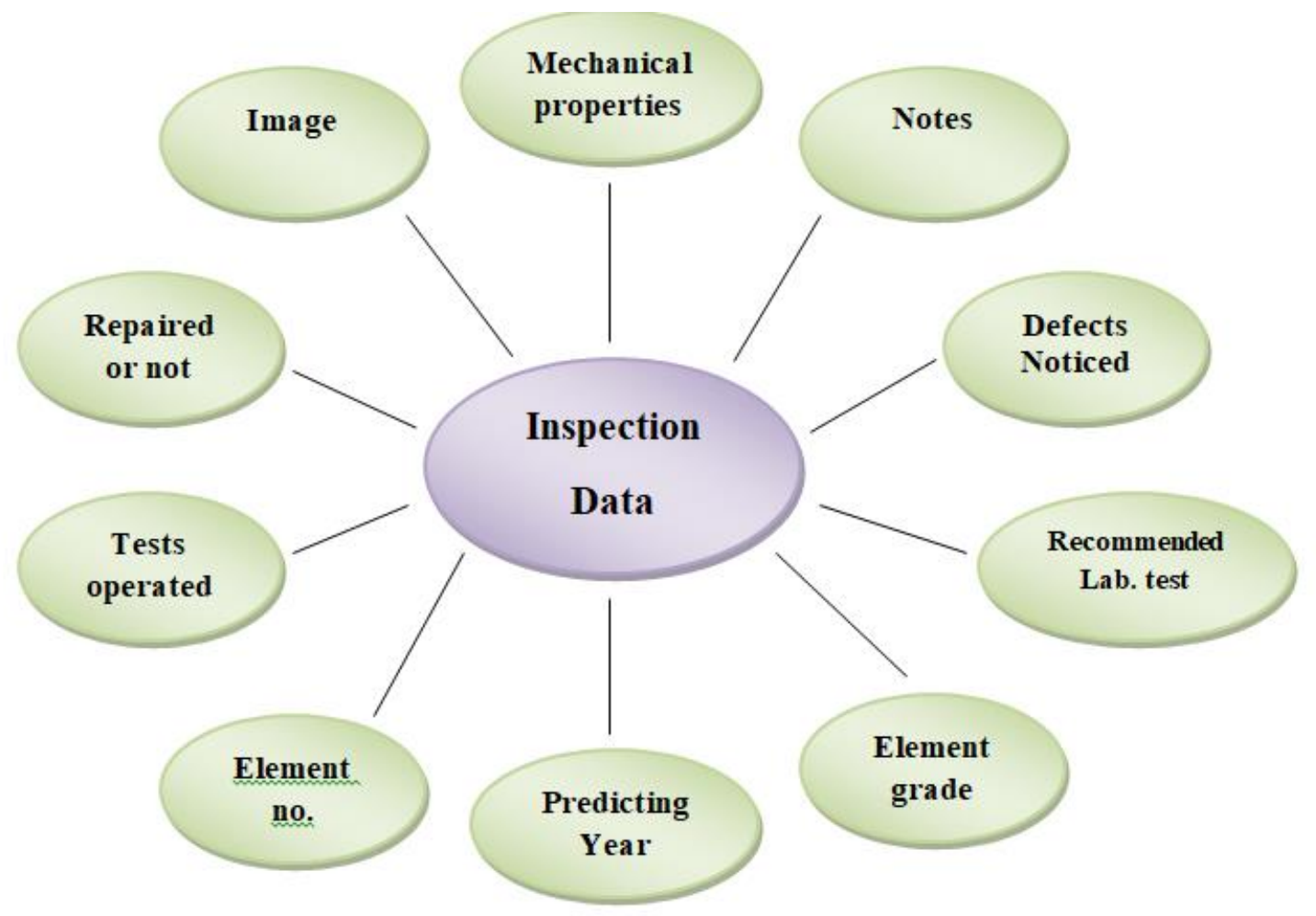

Figure 2: Added Inspection Data Field

\section{Assessment of Defective Structures system}

The condition assessment accurately evaluates the effect of building elements with these defects. Based on the current condition assessment, the element-dependent deterioration model of the framework predicts the element condition for each year in the planning horizon. Depending on the current condition assessment, the proposed deterioration model predicts the future deterioration of the elements for each year in the building life cycle. 
As follows, the present and the future deteriorations are monitored by the proposed framework. A prototype system was developed depend on building elements deficiencies it can be used to manage the buildings and rehabilitation purposes. It has modules for condition assessment and deterioration prediction.

\section{Condition Assessment Model}

To verify and test the suggested methodology, a real model (case study) was chosen in order to apply the proposed method. The model is a building consisting of a ground and four typical floors. It indicates various damage features. After 16 years of its construction an assessment committee was formed to evaluate the structural performance of it and to decide the deserved rehabilitation technique.

After getting condition data from inspection, the common process of condition assessment is as following:

(1) Calculate the deterioration index of each element.

(2) The purpose of a condition assessment is to determine accurate deterioration indices (DIs) for all elements in the building being assessed. Calculate the damage indices for each category (Slabs, Beams, and Columns). Each element of the building is assigned as a set of four states (satisfactory, Fair, Poor, and serious conditions).

(4) Set a respective weight for each element in the building.

(5) Calculate the columns, beams and slabs degradation indices and overall building condition.

\section{Deterioration Prediction Model}

To predict the deterioration of the element, its probability of transition from one condition state to another should be accumulated over discrete time intervals The most commonly used stochastic technique for infrastructure deterioration is the Markov chain model (Abdelalim, 2019).

In this paper, Markov chains were applied to predict the state of each element in the building. Autodesk Revit does not currently have the built in capacity for modeling damage, Nevertheless, developed a method to use existing tools in Revit to accomplish the major objectives in modeling a building and documenting damage information from building inspections. Key requirements for modeling damage include:

- Ability to reduce the mechanical properties of an element.

- Ability to document the location of damaged element.

The objective of BIM condition assessment tool is to develop a digital image database of a building inspection. These images will show elements at various stages of deterioration. Different states can be added to the system along the time and stored in both Excel and Revit parameters. The color coding of the structural elements is displayed in the building's model based on their stages, stage one with green color, 
stage two with yellow color, stage three with orange color and stage four with red color, as shown in figure 3 .

The images from the inspection for each element can be stored to the Revit model. The current image of the selected element describes the element status at the time of the inspection. Therefore, the image is assigned to specified inspection record. Also it can be stored more than one image for specific element.

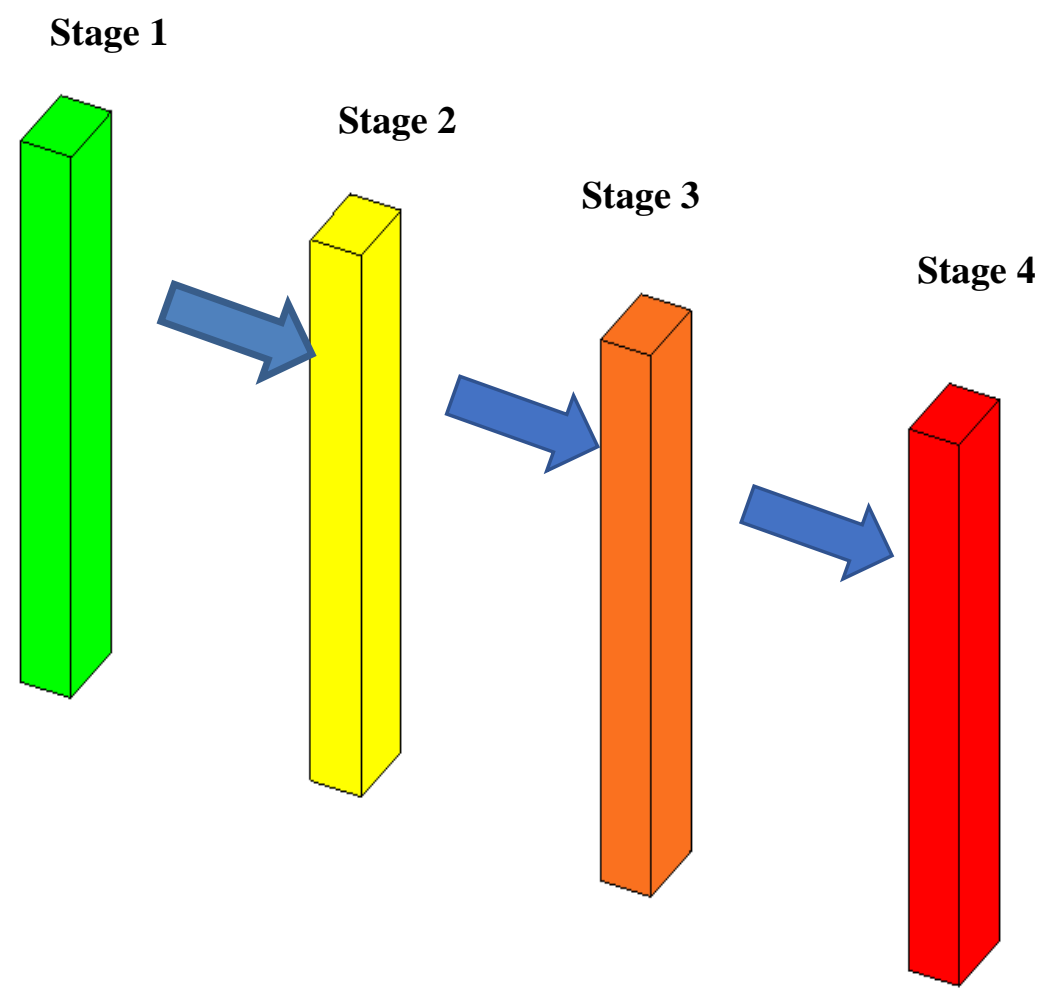

Figure 3: The Four Stages of the Element

\section{Updating the Model Information Using Dynamo Studio}

In this paper condition assessment model and prediction model are developed by using Excel sheets. These sheets can be linked to the Revit model by dynamo studio. Dynamo Studio is a visual programming environment that enables users to explore parametric conceptual designs and automate tasks (Autodesk, 2020).

Integrate automation into the BIM (Building Information Modeling) process, extend designs into interoperable workflows for documentation, coordination, analysis and write code using a simple and powerful scripting interface. Linked Data can include building parameters and inspection data that have been assigned to the elements of the building, script is shown in Figure 4. 
The following section demonstrates the updating of the Revit model data from Excel output for each year during building life cycle. Physical properties after deterioration (compressive strength $f_{c u}$ and area of reinforcement $A_{s}$ ), Degradation index of each element, D.I of slabs, D.I of beams, D.I of columns and over all D.I of the building are the updating data. It can be done by running the dynamo script at each year using running interface in figure 5.

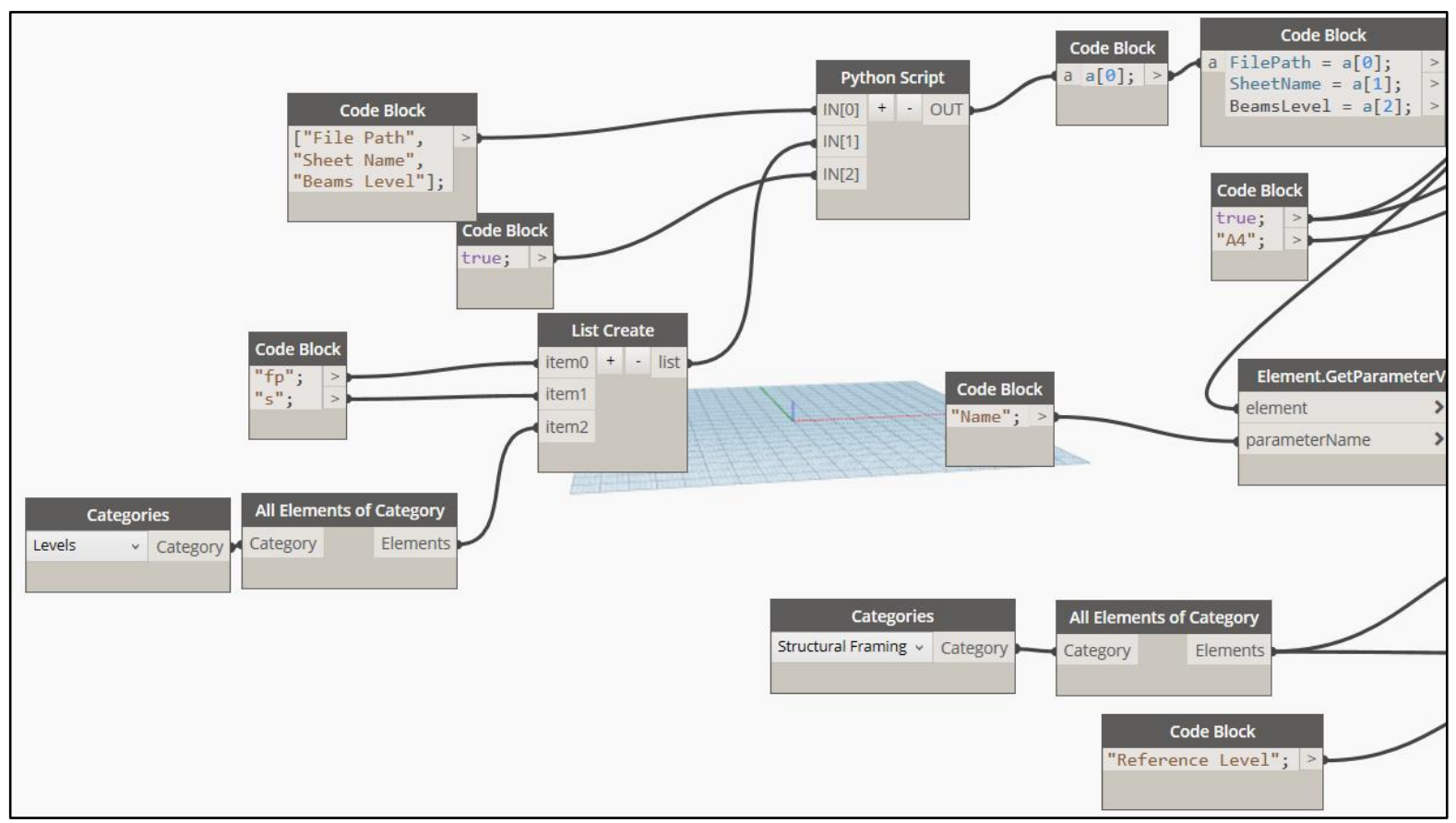

Figure 4: Dynamo Script

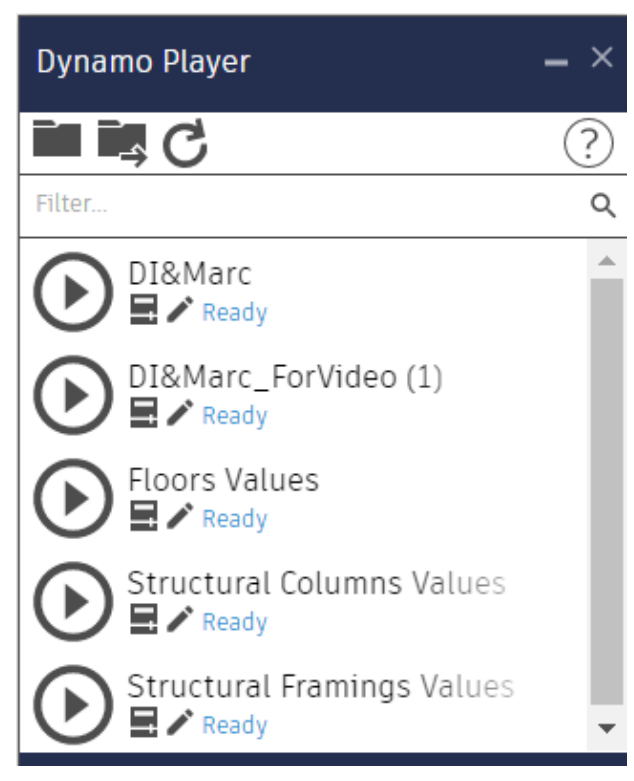

Figure 5: Dynamo Running Interface 
Element deterioration stages can be shown on the model during building life cycle. The first stage started to appear in year 4 , followed by the second stage in year 7 , the third stage in year 8, and finally the fourth stage in year 12.In figure 6 the building deterioration modeling was done so that the condition of each element is seen every year during the building's life cycle.

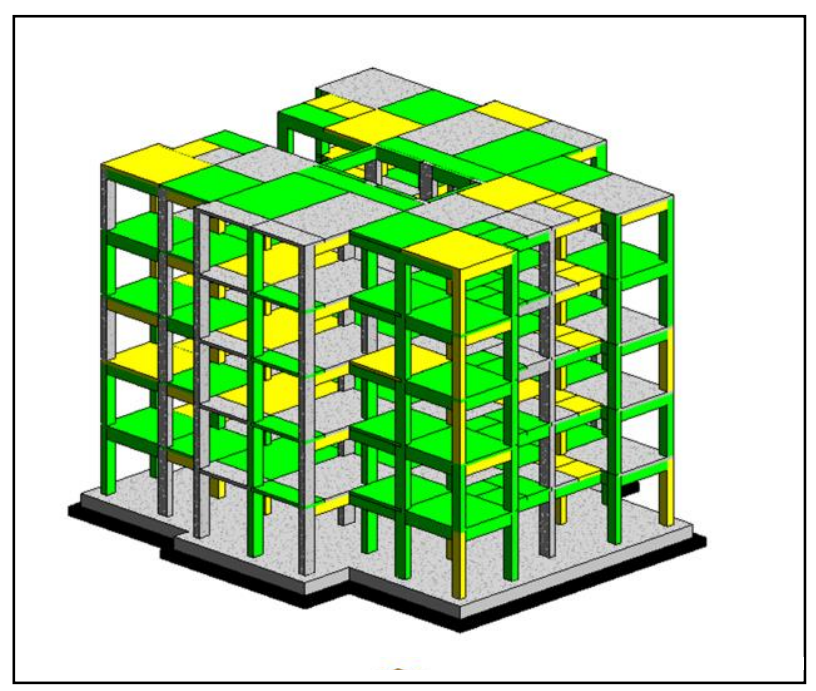

Year10

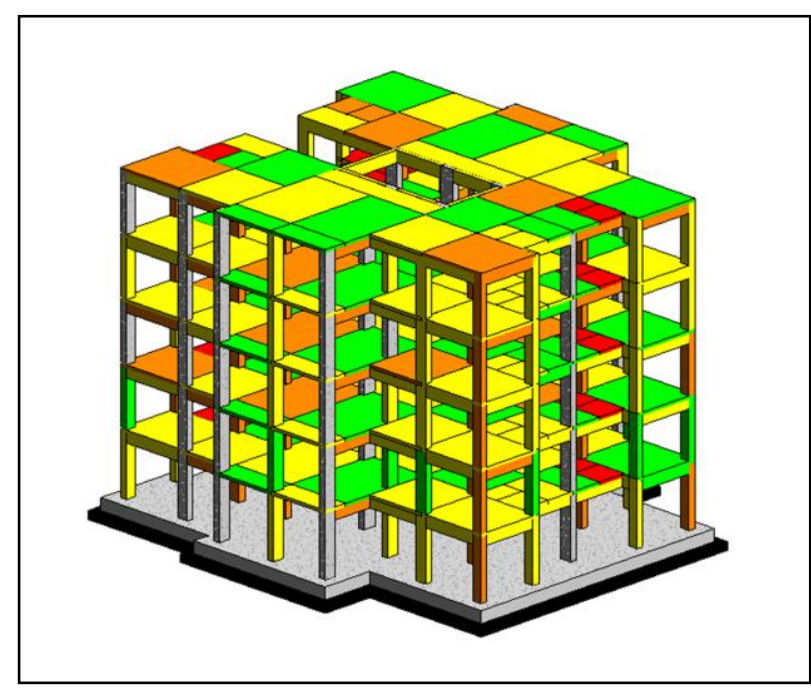

Year 15

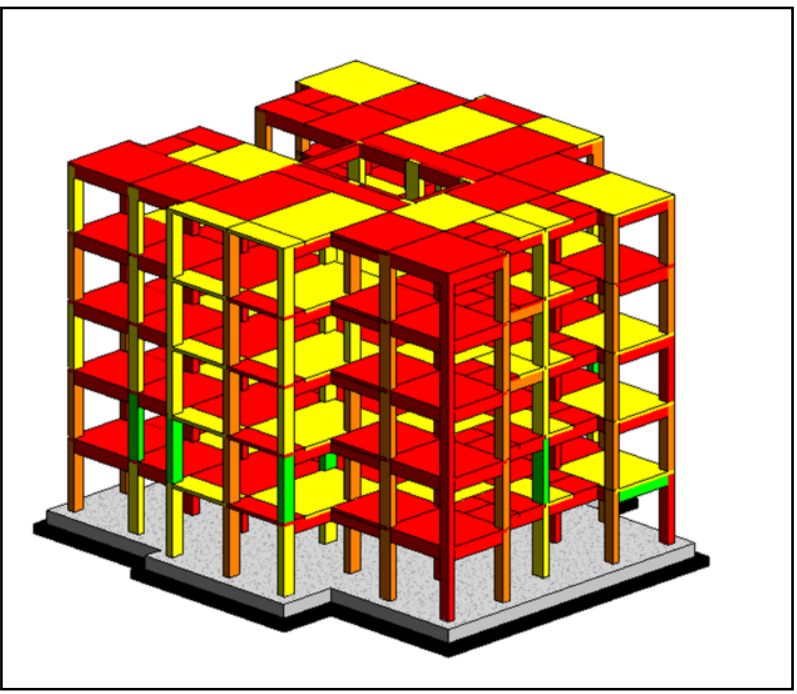

Year 25

Figure 6: 3D Deterioration Model

\section{Integrating BIM with Building Management}

The case study described herein shows that the application of BIM to the operation and maintenance stages of building's service life of the building. In this research, the 
ability for BIM to facilitate the building inspection, evaluation and predicting building condition processes has been demonstrated. During an inspection BIM can offer benefits to building inspectors as a graphical tool in its level of detail and photorealistic rendering capabilities.

The prototype's demonstrated the following benefits:

a. Provide an efficient and automated approach to field data collection, including pictures;

b. Incorporate digital drawings as the basis for data storage and review;

d. Facilitate better resolution of priorities across the data.

The developed framework is expected to help re-engineer the traditional processes for the condition assessment of building infrastructure as well as the decision-making process for maintenance program.

\section{Conclusion}

1. BIM in building management could be favorable by its proven ability to facilitate the inspection and evaluation processes, which could ultimately result in a more automated practice. Utilize maintenance data to minimize inspection effort and prioritize inspection tasks.

2. BIM provide users with several advantages such as faster system, accurate and well organized data collection. This will help decision-makers to have their quick responses toward reported defects.

3. Documenting the deterioration model in the inspection can be significant in determining the structural adequacy of a building element and can affect the guidelines for determining the maintenance time of the current building.

\section{Recommendation for Future Work}

Several potential improvements can be incorporated into the developed condition assessment framework presented in this study, and other areas of research related to the developed system can be explored:

1. Using 3-D laser scanning technology for the BIM modeling of buildings on a network level using point cloud data.

2. Software development in BIM to provide standard, built-in tools for modeling damage on elements and capturing information from an inspection.

3. Interoperability between BIM platforms and industry standard structural analysis, finite element software programs. 


\section{References}

(1) Abdelalim, A. M. (2019) 'A novel diagnostic prognostic approach for rehabilitated RC structures based on integrated probabilistic deterioration models', International Journal of Decision Sciences, Risk and Management. Inderscience Enterprises Ltd, 8(3), pp. 119-134.

(2) Che-Ani, a. i. et al. (2017) 'A review of building information modelling (bim)-based building condition assessment concept', malaysian construction research journal (mcrj), p. 85.

(3) McGuire, B.M., 2014. Using Building Information Modeling to Track and Assess the Structural Condition of Bridges. Degree of Master in Science, Colorado State University.

(4)Autodesk,2020.https://www.autodesk.com/products/dynamostudio/overview?plc=D YNSTD\&term $=1-$ YEAR\&support=ADVANCED\&quantity $=1$

(5) Construction, M. H. (2012) 'The business value of BIM for infrastructure: Addressing America's infrastructure challenges with collaboration and technology', Smart Market Report, p. 1.

(6) Meadati, P. (2009) 'BIM extension into later stages of project life cycle', in Associated Schools of Construction 45th Annual International Conference, pp. 121-129.

(7) Tang, P. and Akinci, B. (2012) 'Formalization of workflows for extracting bridge surveying goals from laser-scanned data', Automation in Construction. Elsevier, 22, pp. 306-319. 